\title{
Numerical study of a hollow cathode neutraliser by means of a zero-dimensional plasma model
}

\author{
Antonio Gurciullo, Andrea Lucca Fabris ${ }^{\dagger}$ \\ Surrey Space Centre, University of Surrey, Guildford, GU2 7XH, United Kingdom \\ and \\ Thomas Potterton ${ }^{\ddagger}$ \\ Surrey Satellite Technology Ltd, Guildford, GU2 7YE, United Kingdom
}

April 21, 2020

\begin{abstract}
A zero-dimension plasma model of thermionic hollow cathodes is here derived. The equations of conservation of mass, current and power are solved within the insert and orifice regions. The model introduces some elements of novelty compared to prior literature such as the inclusion of the electron current collected on the orifice lateral walls, and ion and electron currents collected on the orifice plate walls in the overall balance equations. Estimation of the power deposited on walls due to electron and ion bombardment in both insert and orifice regions are performed, thus, enabling comparisons among different geometrical configurations and operating regimes. The model results are compared against published experimental data and a thorough investigation of the model response to variation of geometrical and operational condition of a sample cathode are presented. A power budget, which includes power consumption and power deposition, of a sample thermionic cathode is also discussed.
\end{abstract}

\section{Nomenclature}

\section{Roman Symbols}

$A_{\text {in }} \quad$ Insert region cross section

$A_{\text {in,lat } \quad \text { Insert lateral surface }}$

$A_{o} \quad$ Orifice cross section, $=\pi r_{o}^{2}$

${ }^{*}$ PhD Student at University of Surrey. Present position: Plasma Physics Research Engineer, Exotrail, Massy, 91300, France, antonio.gurciullo@exotrail.com.

$\dagger$ Lecturer in Electric Propulsion, a.luccafabris@surrey.ac.uk.

$\ddagger$ Propulsion Manager, t.potterton@sstl.co.uk 


\begin{tabular}{|c|c|}
\hline$A_{o}$ & Orifice cross section \\
\hline$A_{o, l a t}$ & Orifice lateral surface \\
\hline$A_{\text {plate }}$ & Plate surface \\
\hline$E_{c}$ & Electric field at the cathode sheath \\
\hline$E_{i z}$ & First ionisation energy \\
\hline$I_{e, i n}^{e m}$ & Field-enhanced thermionic electric current \\
\hline$I_{e, i n}^{\text {lateral }}$ & Random electron current on the lateral insert surface \\
\hline$I_{e, i n-o}$ & Electron current flowing from the insert toward the orifice \\
\hline$I_{e, \text { in }}^{\text {plate }}$ & Electron current on the plate surface \\
\hline$I_{e, o}^{e x t r}$ & Extracted electron current \\
\hline$I_{e, o}^{\text {lateral }}$ & Electron current on the lateral surface of the orifice \\
\hline$I_{i, \text { in }}^{\text {lateral }}$ & Ion current on the lateral insert surface \\
\hline$I_{i, i n}^{\text {plate }}$ & Ion current on the plate surface \\
\hline$I_{i, i n}^{u p}$ & Random ion current flowing upstream the insert volume \\
\hline$I_{i, o}^{e x t r}$ & Extracted ion current \\
\hline$I_{i, o-i n}$ & Ion current flowing from the orifice toward the insert \\
\hline$I_{i, o}^{\text {lateral }}$ & Ion current on the lateral surface of the orifice \\
\hline$K_{e x, o}$ & Excitation rate constant \\
\hline$K_{i z, i n}$ & Ionisation rate in the insert region \\
\hline$K_{i z, o}$ & Ionisation rate in the orifice region \\
\hline$L_{i n}$ & Axial length of the cathode insert \\
\hline$L_{o}$ & Axial length of the cathode orifice \\
\hline$\dot{m}_{\mathrm{Xe}}$ & xenon mass flow rate \\
\hline$m_{\mathrm{Xe}}$ & xenon mass \\
\hline$n_{e, i n}$ & Electron density in the cathode insert volume \\
\hline$n_{e, o}$ & Electron density in the cathode orifice volume \\
\hline$N_{i, i n}$ & Number of ions in the cathode insert volume \\
\hline
\end{tabular}




\begin{tabular}{|c|c|}
\hline$N_{i, o}$ & Number of ions in the cathode orifice volume \\
\hline$n_{n}$ & Neutral density \\
\hline$n_{n, i n}$ & Neutral density in the cathode insert volume \\
\hline$n_{n, o}$ & Neutral density in the cathode orifice volume \\
\hline$P_{d s}$ & Double sheath power \\
\hline$p_{n}$ & Neutral pressure \\
\hline$p_{n, i n}$ & Neutral pressure in the insert volume \\
\hline$p_{n, o}$ & Neutral pressure in the orifice volume \\
\hline$P_{s}$ & Sheat power \\
\hline$P_{t o t}$ & Total power \\
\hline$P_{\omega, \text { in }}$ & Joule heating in the insert plasma \\
\hline$P_{\omega, o}$ & Joule heating in the orifice plasma \\
\hline$Q$ & Volumetric flow rate \\
\hline$r_{i n}$ & Inner radius of the cathode insert \\
\hline$r_{o}$ & Inner radius of the cathode orifice \\
\hline$R_{\Omega, i n}$ & Plasma resistance in the insert region \\
\hline$R_{\Omega, o}$ & Plasma resistance in the orifice region \\
\hline$T_{e}$ & Electron temperature \\
\hline$T_{e, i n}$ & Electron temperature in the cathode insert volume \\
\hline$T_{e, o}$ & Electron temperature in the cathode orifice volume \\
\hline$T_{i}$ & Ion temperature \\
\hline$T_{i, i n}$ & Ion temperature in the cathode insert volume \\
\hline$T_{i, o}$ & Ion temperature in the cathode orifice volume \\
\hline$T_{n}$ & Neutral temperature \\
\hline$T_{n, i n}$ & Neutral temperature in the cathode insert volume \\
\hline$T_{n, o}$ & Neutral temperature in the cathode orifice volume \\
\hline$T_{r}$ & Adimensional variable, $=T_{n, \text { in }}[\mathrm{K}] /(289.7 \mathrm{~K})$ \\
\hline$T_{w}$ & Wall temperature \\
\hline
\end{tabular}




$\begin{array}{ll}T_{w, i n} & \text { Insert temperature } \\ T_{w, o} & \text { Orifice wall temperature } \\ U_{e x} & \text { Average energy loss per excitation } \\ v_{B, i n} & \text { Bohm velocity in the insert region } \\ v_{B, o} & \text { Bohm velocity in the orifice region } \\ V_{d s} & \text { Potential drop across the double sheath } \\ \bar{v}_{e, o} & \text { Average electron velocity in the orifice volume } \\ \bar{v}_{i, i n} & \text { Average ion speed in the insert region } \\ V_{i n} & \text { Volume of the insert region } \\ \bar{v}_{i, o} & \text { Average ion speed in the orifice region } \\ V_{o} & \text { Volume of the orifice region }\end{array}$

\section{Greek Symbols}

$\begin{array}{ll}\gamma & \text { Specific heat ratio } \\ \zeta & \text { Gas viscosity } \\ \eta & \text { Plasma resistivity } \\ \lambda_{n} & \text { Mean free path of neutrals } \\ \nu_{e i} & \text { Frequency of electron-ion collisions } \\ \nu_{e n} & \text { Frequency of electron-neutral collisions } \\ \sigma_{e n} & \text { Electron-neutral collision cross section } \\ \sigma_{e x} & \text { Excitation collision cross section } \\ \phi_{e f f} & \text { Effective work function } \\ \phi_{s} & \text { Sheath potential drop } \\ \phi_{w f} & \text { Work function }\end{array}$




\section{Introduction}

IOLLOW cathode neutralisers (HCN) are essential components for the operHation of some plasma thrusters such as Gridded Ion Engines [1], Hall Effect Thrusters [2] and Quad Confinement Thrusters [3], which require an electron source to start and sustain the plasma discharge, and neutralise the ejected ion beam. Since hollow cathodes are widely employed in laboratory test campaigns and space missions, a significant amount of research effort has been directed toward the investigation of computational tools to predict their performance and to support their design at various ranges of power and emitted current.

These computational tools include from 0-D to 2-D plasma models [4-18] whose input parameters generally are geometry of the cathode, gas properties, some plasma properties (assumed or experimentally measured), operating conditions and, in some cases, free parameters that allow the model results to be matched with the experimental outcomes. Depending on the approach, the models can predict plasma density, electron temperature, plasma potential, neutral density, power deposition on the internal surface of the cathode, emitter temperature and cathode lifetime. The reader may find a comprehensive critical review signed by Wordingham et al. [19] that covers most of the zero-dimensional models proposed until today.

The model presented in this paper presents a similar approach to that proposed by Domonkos [11] and Albertoni et al. [16], in which the equations of conservation of mass, current and power are solved within two cathode plasma regions, namely the insert and orifice regions. At the same time, the model introduces some elements of novelty compared to prior literature such as the inclusion of the electron current collected on the orifice lateral walls, and ion and electron currents collected on the orifice plate walls in the overall balance equations. The model estimates the power deposited on walls due to electron and ion bombardment in both insert and orifice regions, enabling comparisons among different geometrical configurations and operating regimes. The model is intended for being coupled with a thermal solver to provide a self-consistent plasma-thermal solution in which only the operational parameters are the input variables. However, this activity is out of the scope of this research paper.

The following sections will describe the physical-mathematical framework along with the computational cycle (Section 2), and results of simulations for various operating conditions and cathode configurations (Section 3).

\section{Plasma model of Hollow Cathode Neutralisers}

\subsection{Overview of the Hollow Cathode Plasma Model}

The hollow cathode plasma volume is divided into two regions, the insert region and the orifice region, as depicted in Figure 1. In each region a set of equations of conservation is solved iteratively until the solutions of the unknown parameters converge. The unknown parameters are the electron temperature 


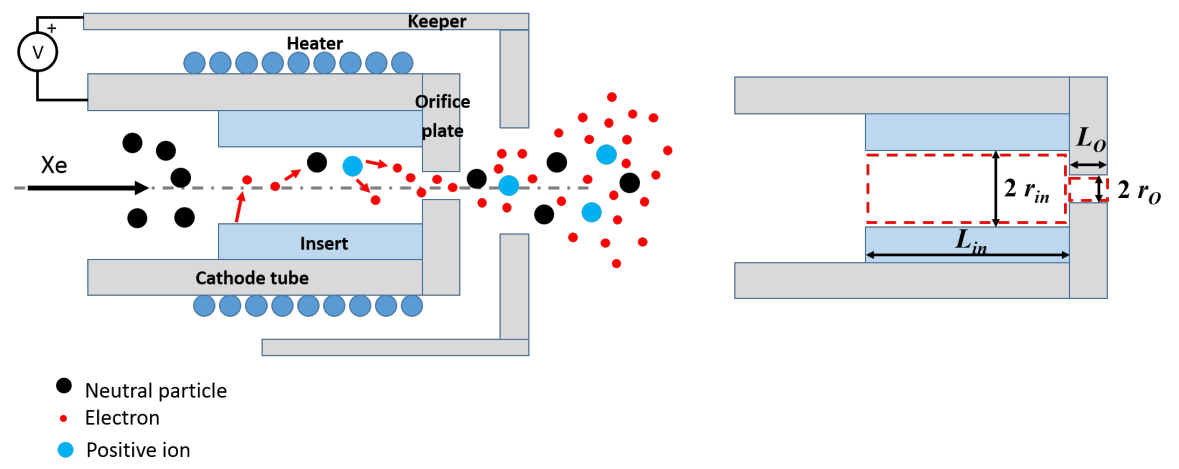

Figure 1: Schematic of the hollow cathode regions.

$\left(T_{e, o}, T_{e, i n}\right)$, plasma density $\left(n_{e, o}, n_{e, i n}\right)$ and the sheath potential $\left(\phi_{s}\right)$, where the subscripts "o" and "in" stand for "orifice" and "insert", respectively. The plasma properties are averaged within the cathode regions (0-D model). The wall temperature is assumed fixed. The working temperature resides in a specific temperature range, outside of which the model does not converge. In other words, the wall temperature is a free parameter and it can be selected either manually or by coupling the plasma model with a thermal model. An initial guess of the wall temperature is computed by solving the Richardson-Dushman equation (presented in later sections, in equation (27)) for a selected geometry and material of the emitter at an emitted electron current approximately equal to the discharge current. This temperature selection process requires iterations since, as explained below, the effective working function of the material is modified by the presence of an electric field (thus, by the Schottky effect [20]) which is calculated within the model.

For the sake of simplicity, the plasma is formed by three species, namely neutrals, singly-charged ions and electrons. The ion-neutral collisions allow the heavy particles (ions and neutrals) to be in thermal equilibrium between each other and their temperature is assumed to be equal to the temperature of the wall, i.e. $T_{i}=T_{n}=T_{w}$, where $T_{i}, T_{n}$ and $T_{w}$ are the ion, neutral and wall temperatures, respectively. Both assumptions are questionable, since it has been experimentally observed by Williams et al. [21] that neutrals may have a temperature much higher than the wall temperature and 2-D models [22] have predicted that neutrals and ions are not in thermal equilibrium inside the cathode, although they have similar peak temperatures. Sary et al. [22] explains that ion-neutral collisions are not frequent enough (collision frequency: $10^{6} \mathrm{~s}^{-1}$ ) to favour a thermal equilibrium between the heavy particles, whereas electron-ion and electron-neutral collisions have a much higher collision frequency $\left(10^{9} \mathrm{~s}^{-1}\right)$. Therefore, despite the mass ratio between the colliding particles is more favourable among ion-neutral collisions, ion and neutrals are mainly heated via collisions with electrons. Nevertheless, in 0-D models the typically 
accepted assumptions are either $T_{n}=T_{i}=T_{w}$ or $2 T_{w} \leq T_{n}=T_{i} \leq 4 T_{w}$. In our case, the first assumption is adopted.

Some approaches take into account an effective length of the emitter [19], also known as attachment length, from which the electrons are thermionically emitted. The effective length has been experimentally observed [13], however a consensus on its modeling has not been reached yet. As consequence, this length has been a free parameter in many models. A more rigorous approach would require to divide the emitter in smaller domains in order to simulate the temperature gradient observed in experiments and, possibly, correctly estimate the size of the attachment length. Since more complex models are prone to uncertainty in any case, the simplest approach has been followed, in which the temperature gradient in the emitter material is neglected and the attachment length is assumed equal to the overall emitter axial length.

For all the simulations described in this paper, the working gas is xenon whose physical characteristics are well known.

\subsection{Neutral flow}

In this section, the equations governing the neutral flow are described. First, let us consider the orifice region. The orifice is a restriction where the pressure upstream is higher than the pressure downstream and, typically, the flow is treated as chocked [11]. The neutral density at the exit of the orifice is

$$
n_{n, o}=\frac{\dot{m}_{\mathrm{Xe}}}{M_{\mathrm{Xe}} \pi r_{o}^{2} \sqrt{\gamma R T_{w, o}}},
$$

where $\dot{m}_{\mathrm{Xe}}$ is the xenon mass flow rate, $M_{\mathrm{Xe}}$ is the xenon atomic mass, $r_{o}$ is the orifice radius, $\gamma$ is the specific heat ratio of xenon, $R$ is the specific gas constant in $\mathrm{J} /(\mathrm{K} \mathrm{kg})$ and $T_{w, o}$ is the orifice wall temperature. The associated sonic pressure at the orifice is

$$
p_{\text {sonic }, o}=p_{n, o}=\frac{\dot{m}_{\mathrm{Xe}} R T_{w, o}}{\pi r_{o}^{2} \sqrt{\gamma R T_{w, o}}} .
$$

Since within the insert region neutral-neutral collisions rather than collisions with the walls govern the neutral flow motion, the pressure through the cathode tube in the insert region is approximated by the Poiseuille law [23]. This is true if the Knudsen number is sufficiently low:

$$
\mathrm{Kn}=\frac{\lambda_{n}}{2 r_{i n}},
$$

where $r_{i n}$ is the inner radius of the emitter and [23]

$$
\lambda_{n}=\frac{1}{\sqrt{2} n_{n, i n} \pi \delta^{2}}
$$

is the mean free path of neutrals in a homogeneous Maxwellian gas. The parameter $\delta$ is the atomic diameter of the gas particle, for xenon it is $\delta=4.87 \cdot 10^{-10} \mathrm{~m}$ 
(from Ref. [24] p.46). The neutral pressure in the insert region is related to the pressure and temperature of the gas as follows ( [13], Appendix B)

$$
n_{n, i n}\left[\mathrm{~m}^{-3}\right]=9.65 \cdot 10^{24} \frac{p_{n, i n},[\text { Torr }]}{T_{n, i n}[\mathrm{~K}]},
$$

where $p_{n, i n}$ and $T_{n, i n}$ are the pressure and the temperature of the neutrals in the insert region, respectively. In a typical hollow cathode neutraliser, the neutral pressure in the insert region is in the range 1-10 Torr [13]. Assuming a neutral temperature equal to that of the wall, about $1300 \mathrm{~K}$ for a $\mathrm{BaO}$ insert, and an insert of inner radius $r_{i n}=0.5 \mathrm{~mm}$, the Knudsen number is $\mathrm{Kn} \approx 0.13$. A flow can be treated as a continuum if $\mathrm{Kn}<0.01$ and falls into the free-molecular regime if $\mathrm{Kn}>1$. In this case, at $\mathrm{Kn} \approx 0.13$, the flow is in a transitional combination of continuous and molecular flow. Thus, it is a bit of a stretch to describe the flow by the Poiseuille's law, however this approach has been adopted in various hollow cathode models and accepted by the scientific community [25]. The neutral pressure inside the insert region is [13]

$$
p_{n, i n}=\sqrt{p_{n, o}^{2}+\frac{0.78 Q \zeta T_{r} L_{i n}}{\left(2 r_{i n}\right)^{4}}},
$$

where $p_{n, o}$ is the neutral pressure in the orifice, $Q$ is the xenon flow rate in sccm, $T_{r}=T_{n, i n}[\mathrm{~K}] /(289.7 \mathrm{~K}), L_{i n}$ is the insert axial length, $r_{i n}$ is the insert inner radius and

$$
\zeta=2.3 \cdot 10^{-5} T_{r}^{\left(0.71+0.29 / T_{r}\right)} \quad \text { for } T_{r}>1
$$

is the viscosity of xenon in $\mathrm{Ns} / \mathrm{m}^{2}$.

\subsection{Orifice plasma model}

The orifice region is delimited by three boundaries, as seen in Figure 2. The boundary shared with the insert region (on the left of the figure) is modelled as a double sheath. The double sheath forms at a constriction (the orifice, in our case) as a consequence of discharge current continuity. As explained by Andrews and Allen [26], at steady state it is required that the electron density inside the constriction is greater than that outside (the insert region, in our case) in order to maintain the total discharge current continuous through the constriction. In the orifice, the ratio of ion loss rate to the creation rate is higher than that in the insert region, due to a higher surface-to-volume ratio of the orifice region compared to that of the insert region. Thus, to maintain the plasma quasi-neutral in the orifice, the electrons flowing from the insert need to have a higher energy in order to sustain a high ionisation rate. This surplus of energy is provided by the acceleration the electrons experience while passing through the double sheath. The double sheath can be imagined as a boundary on which the space charge facing the insert volume is negative and the side facing the orifice has a positive space charge. Thus, electrons are allowed to pass from the insert region toward the orifice, while they are reflected back if 


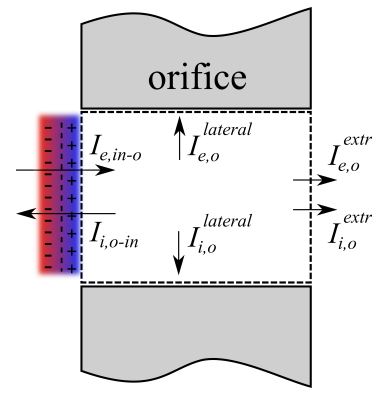

Figure 2: Current continuity in the orifice region.

coming from the orifice volume. Similarly, the ions are reflected back if coming from the insert side and are allowed to pass though the double sheath when coming from the orifice volume. Although Andrews and Allen [26] found that a double sheath at a constriction always bulges out, the voltage across the double sheath can be approximated planar for small geometries and the potential across is, as derived by Crawford and Freeston [27] cited in Carpacci et al. [10],

$$
V_{\mathrm{ds}}=\left[\frac{9\left(I_{i, o-i n}+I_{e, i n-o}\right) k_{B} T_{e, i n}}{7.5 A_{o} n_{e, i n} e^{2}} \sqrt{\frac{m_{e}}{2 e}}\right]^{2 / 3},
$$

where $I_{i, o-i n}$ is the ion current flowing from the orifice toward the insert, $I_{e, i n-o}$ is the electron current flowing from the insert to the orifice, $T_{e, i n}$ is the electron temperature in the insert region, $A_{o}=\pi r_{o}^{2}$ is the orifice cross section, $n_{e, i n}$ is the electron density in the insert, $e$ is the elementary charge and $m_{e}$ is the electron mass.

In addition to the double sheath, the ions are allowed to leave the orifice plasma volume through the lateral wall of the orifice and downstream of the orifice. Similarly, electrons flow out through the lateral walls and the downstream of the orifice. In the following sections, the equations of conservation solved within the orifice region are presented and discussed.

\subsubsection{Current continuity}

As shown in Figure 2, the sum of the electron and ion currents leaving through the orifice boundaries are, for the current continuity, balanced:

$$
-I_{i, o}^{\text {lateral }}+I_{e, o}^{\text {lateral }}+I_{e, o}^{\text {extr }}-I_{i, o-i n}-I_{e, i n-o}-I_{i, o}^{e x t r}=0,
$$

where the six terms are:

1. the ion current toward the orifice lateral surface, on which presheath and sheath form,

$$
I_{i, o}^{\text {lateral }}=e 0.606 n_{e, o} \sqrt{\frac{e T_{e, o}}{m_{\mathrm{Xe}}}} A_{o, l a t}
$$


where $A_{o, l a t}=2 \pi r_{o} L_{o}$ is the orifice lateral surface. A presheath and sheath are assumed forming on this surface. Ions are accelerated in the presheath and reach the presheath-sheath boundary with a velocity $v_{B, o}=$ $\sqrt{e T_{r, o} / m_{\mathrm{Xe}}}$, known as Bohm velocity, and the factor 0.606 is the ratio of the plasma density at the sheath border to that in the bulk plasma which is calculated from the Boltzmann relation (see Ref. [28] p.169 and p.172).

2. The electron current toward the orifice lateral surface which is dominated by a random electron flux at an average velocity $\bar{v}_{e, o}=\sqrt{8 e T_{e, o} / \pi m_{e}}$ in the assumption of a Maxwellian distribution,

$$
I_{e, o}^{\text {lateral }}=\frac{e}{4} n_{e, o} \sqrt{\frac{8 e T_{e, o}}{\pi m_{e}}} \exp \left(-\frac{\phi_{s}}{T_{e, o}}\right) A_{o, l a t},
$$

where $\phi_{s}$ is the potential drop across the sheath.

3. The ion current leaving through the double sheath, which is modelled as a permeable wall on which presheath and sheath form,

$$
I_{i, o-i n}=e 0.606 n_{e, o} \sqrt{\frac{e T_{e, o}}{m_{\mathrm{Xe}}}} A_{o} .
$$

4. The electron current coming from the insert through the double sheath, $I_{e, i n-o}$, which is computed rearranging the terms in equation (9):

$$
\begin{aligned}
I_{e, \text { in }-o} & =-I_{i, o}^{\text {lateral }}+I_{e, o}^{\text {lateral }}+I_{e, o}^{\text {extr }}-I_{i, o}^{\text {extr }}-I_{i, o-i n}= \\
& =-I_{i, o}^{\text {lateral }}+I_{e, o}^{\text {lateral }}+I_{D}-I_{i, o-i n} .
\end{aligned}
$$

5. The extracted electron current $I_{e, o}^{e x t r}$ which is derived as it follows. Note that the total extracted current is the discharge current $I_{D}$, thus

$$
I_{D}=I_{e, o}^{e x t r}-I_{i, o}^{e x t r}
$$

and, since $I_{D}$ is known (it is one of the fixed values imposed at the beginning of the algorithm), the extracted electron current is readily calculated:

$$
I_{e, o}^{e x t r}=I_{D}+I_{i, o}^{e x t r} .
$$

6. The random ion flux that flows through the orifice exit, assumed a Maxwellian distribution, at ion average velocity $\bar{v}_{i, o}=\sqrt{8 e T_{i, o} / \pi m_{\mathrm{Xe}}}$

$$
I_{i, o}^{e x t r}=\frac{e}{4} n_{e, o} \sqrt{\frac{8 e T_{i, o}}{\pi m_{\mathrm{Xe}}}} A_{o} .
$$

For the sake of completeness, equation (9) should include the contribution of the thermionic electron current from the orifice surface. However, since the aim of this manuscript is to investigate conventional thermionic cathodes whose orifice is made by high-work-function refractory metal, this current contribution is expected to have a negligible impact on the study presented in the discussion section below. 


\subsubsection{Ion mass conservation}

The simulated plasma is at steady-state, which means that the ion production and the incoming ion flux are balanced by the outgoing ion flux. The number of ions created and lost per unit time is

$$
\begin{aligned}
\frac{d N_{i, o}}{d t} & =0= \\
& =\underbrace{V_{o}\left(n_{n, o}-n_{e, o}\right) n_{e, o} K_{i z, o}}_{\text {ionisation }}+ \\
& -\underbrace{0.606 n_{e, o} v_{B, o}\left(A_{o}+A_{o, l a t}\right)}_{\text {lateral walls and double sheath }}+ \\
& -\underbrace{\frac{n_{e, o}}{4} \bar{v}_{i, o} A_{o}}_{\text {orifice exit }} .
\end{aligned}
$$

The first term on the right hand side of equation (17) is the ionisation term, where $V_{o}=\pi r_{o}^{2} L_{o}$ is the volume inside the orifice, $n_{n, o}$ is the neutral density, $n_{e, o}$ is the electron density which, for the quasi-neutrality assumption, is equal to the ion density $n_{i, o}$. The ionisation rate is given by the semi-empirical formula, which is derived from the fitting of the cross section $\sigma_{i z}$ averaged over a Maxwellian distribution times the electron velocity $\bar{v}_{e, o},[13]$

$$
\begin{aligned}
K_{i z, o} & =\left\langle\sigma_{i z} v_{e, o}\right\rangle \approx \\
& \approx\left\langle\sigma_{i z}\right\rangle \bar{v}_{e, o}= \\
& =10^{-20}\left[\left(3.97+0.643 T_{e, o}-0.0368 T_{e, o}^{2}\right) \exp \left(-12.127 / T_{e, o}\right)\right] \sqrt{\frac{8 e T_{e, o}}{\pi m_{e}}},
\end{aligned}
$$

where $\sigma_{i z}$ is the ionisation cross section, $\bar{v}_{e, o}$ is the average electron velocity and $T_{e, o}$ is the electron temperature in the orifice in $\mathrm{eV}$. This equation is valid for $T_{e, o}<5 \mathrm{eV}$. The ionisation term takes into account the depletion of neutrals as newborn ions are created, i.e. the residual neutral density is $\left(n_{n, o}-n_{e, o}\right)$.

The second term on the right hand side is due to the ions leaving the plasma volume through the sheath at the lateral wall and the double sheath. In both cases, a presheath and the Bohm condition - to enter the sheath, ions must have a velocity higher or equal to the Bohm velocity - are imposed (see Ref. [28] p.169 and p.172). The Bohm velocity is $v_{B, o}=\sqrt{e T_{e, o} / m_{\mathrm{Xe}}}$. The orifice lateral surface is $A_{o, l a t}=2 \pi r_{o} L_{o}$ and the orifice cross section is $A_{o}=\pi r_{o}^{2}$.

The last term on the right hand side is the number of ions per unit time leaving the orifice through the exit. This flow is modelled by a random flux at average speed $\bar{v}_{i, o}$.

The unknowns in equation (17) are the orifice plasma density $n_{e, o}$ and the orifice electron temperature $T_{e, o}$. 


\subsubsection{Power balance}

Regarding the power balance, the power absorbed by the plasma is the sum of the Joule heating and the energy per unit time carried by the electrons transported from the insert by convection and accelerated through the double sheath. The power losses include ionisation and excitation collisions, energy per unit time transported by the electrons and ions leaving the plasma boundaries. The power balance is

$$
\begin{aligned}
& \underbrace{R_{\Omega, o} I_{D}^{2}}_{\text {Joule heating }}+\underbrace{I_{e, \text { in-o }}\left(\frac{5}{2} T_{e, i n}+V_{d s}\right)}_{\text {convection }+ \text { double sheath }}= \\
& =\underbrace{V_{o}\left(n_{n, o}-n_{e, o}\right) n_{e, o} K_{i z, o} e E_{i z}}_{\text {ionisation }}+ \\
& +\underbrace{V_{o}\left(n_{n, o}-n_{e, o}\right) n_{e, o} K_{e x, o} e U_{e x}}_{\text {excitation }}+ \\
& +\underbrace{I_{e, o}^{\text {extr }} \frac{5}{2} T_{e, o}}_{\text {extracted electrons }}+ \\
& +\underbrace{I_{i, o}^{\text {extr }} 2 T_{i, o}}_{\text {extracted ions }}+ \\
& +\underbrace{I_{i, o-i n} \frac{T_{e, o}}{2}}_{\text {ions toward insert }}+ \\
& +\underbrace{I_{i, o}^{\text {lateral }}\left(\frac{T_{e, o}}{2}+\phi_{s}\right)}+ \\
& \text { ions on lateral wall } \\
& +\underbrace{I_{e, o}^{\text {lateral }} 2 T_{e, o}}_{\text {electrons on lateral wall }} .
\end{aligned}
$$

Let us comment on the Joule heating term. The resistance of the plasma $R_{\Omega, o}$ takes into consideration electron-ion and electron-neutral collisions [13]:

$$
R_{\Omega, o}=\eta \frac{L_{o}}{A_{o}}
$$

where

$$
\eta=\frac{\nu_{e i}+\nu_{e n}}{\epsilon_{0} \omega_{p}^{2}}=\frac{m_{e}\left(\nu_{e i}+\nu_{e n}\right)}{e^{2} n_{e, o}}
$$

is the plasma resistivity, $\omega_{p}=\sqrt{e^{2} n_{e, o} / \epsilon_{0} / m_{e}}$ is the plasma natural frequency of oscillation. The electron-ion collision frequency is [13]

$$
\nu_{e i}=2.9 \cdot 10^{-12} \frac{n_{e, o} \ln \Lambda}{T_{e, o}^{1.5}},
$$


where $\ln \Lambda$ is the Coulomb logarithm

$$
\ln \Lambda=23-\frac{1}{2} \ln \left(\frac{10^{-6} n_{e, o}}{T_{e, o}^{3}}\right) .
$$

The electron-neutral collision frequency is

$$
\nu_{e n}=\sigma_{e n}\left(n_{n, o}-n_{e, o}\right) \sqrt{\frac{8 e T_{e, o}}{\pi m_{e}}},
$$

where $\sigma_{e n}$ is the electron-neutral collision cross section, which for xenon is found by a numerical fit to the electron-neutral scattering cross section averaged over a Maxwellian electron distribution [14]:

$$
\sigma_{e n}=6.6 \cdot 10^{-19}\left[\frac{\frac{T_{e, o}}{4}-0.1}{1+\left(\frac{T_{e, o}}{4}\right)^{1.6}}\right],
$$

where the electron temperature is expressed in $\mathrm{eV}$. The main direction of electron flow is along the orifice channel, thus the ratio $L_{o} / A_{o}$ in equation (20), and the current is approximated by the total extracted current $I_{D}$.

The second term on the left hand side of equation (19) is the power carried by the electrons that come from the insert due to convection (energy term $5 / 2 T_{e, \text { in }}$ ) and are accelerated by the double sheath potential drop (energy term $V_{d s}$ ). The convection term $5 / 2 T_{e}$ comes from the sum of the internal energy $3 / 2 T_{e}$ carried by the electrons during their motion and the mechanical work $T_{e}$ done by the electrons as they move. These energy terms are obtained by deriving the energy transport equation, that is by integrating the Boltzmann equation multiplied by the particle kinetic energy over the velocity space, see Ref. [29] pp.204-209 for a more detailed discussion.

The first term on the right hand side of equation (19) is the power loss due to ionisation in the orifice volume $V_{o}$. This term takes into account the reduced number of neutrals due to ionisations, thus the residual neutral density is $\left(n_{n, o}-n_{e, o}\right)$. The ionisation energy for xenon is $E_{i z}=12.13 \mathrm{eV}$ and the ionisation rate $K_{i z, o}$ is that in equation (18).

The second term on the right hand side of equation (19) is the power loss due to excitation collisions between electrons and neutrals. The average energy loss per excitation is $U_{e x}$ which is $10 \mathrm{eV}$ for xenon [7]. For xenon, the Maxwellian-weighted excitation rate constant as function of the electron temperature is $[13,30]$

$$
\begin{aligned}
K_{e x, o} & =\left\langle\sigma_{e x} v_{e, o}\right\rangle \approx \\
& \approx\left\langle\sigma_{e x}\right\rangle \bar{v}_{e, o}= \\
& =1.93 \cdot 10^{-19} \frac{\exp \left(-11.6 / T_{e, o}\right)}{\sqrt{T_{e, o}}} \sqrt{\frac{8 e T_{e, o}}{\pi m_{e}}} .
\end{aligned}
$$

Electrons and ions exit the orifice downstream with energies equal to $5 / 2 T_{e, o}$ and $2 T_{i, o}$, respectively, as seen from the third and fourth terms on the right 
hand side of equation (19). Electrons are assumed to travel along the orifice by forced convection, that is the electrons are extracted from the cathode through the orifice exit while colder electrons coming from the insert replace those hotter extracted electrons. This explains the energy term $5 / 2 T_{e, o}$ for the extracted electrons and the energy term $5 / 2 T_{e, i n}$ for the electrons convected from the insert. On the other hand, the ion temperature is assumed constant within both volumes, thus, the convection assumption is not valid for the ion species. Instead, the ions exiting the orifice are assumed to carry an average energy of Maxwellian particles crossing the boundary surface $A_{o}$, thus the energy term $2 T_{i, o}$.

The fifth term on the right hand side of equation (19) is the energy per unit time carried by the ions travelling toward the insert. The double sheath is modelled as a permeable wall, where a presheath and a collisionless sheath are present. The orifice region terminates at the presheath-sheath edge of the double sheath region, thus, within the orifice volume, the ions acquire an energy equal to $0.5 T_{e, o}$ (see Ref. [28] p.171) before entering the double sheath.

The sixth term accounts for the energy loss due to ions leaving the plasma toward the lateral orifice wall. Here, a presheath and a sheath are present and the total energy gained by the ions is $\left(0.5 T_{e, o}+\phi_{s}\right)$, where $\phi_{s}$ is the plasma sheath potential drop.

The last term is the energy per unit time lost due to the energetic electrons able to cross the lateral wall sheath. At the wall, they carry an energy equal to $2 T_{e, o}$ (see Ref. [28] p.332).

The unknowns in equation (19) are the orifice plasma density $n_{e, o}$, the orifice electron temperature $T_{e, o}$, insert electron temperature $T_{e, i n}$ and the sheath potential drop $\phi_{s}$.

\subsection{Insert plasma model}

The insert region is delimited by four boundaries, as seen in Figure 3. On the right of the insert region there is the boundary shared with the orifice, i.e. the double sheath, and the orifice plate. It is assumed that both ions and electrons are lost on the orifice plate. On the other hand, it is assumed that only ions leave the insert region by passing through the upstream boundary (on the left of the insert region). The lateral boundary is delimited by the thermionic emitter. Here, thermionically emitted electrons enter the plasma by passing through the sheath potential $\phi_{s}$. It is assumed that all the materials of the insert and orifice walls are conductive, thus sharing the same plasma sheath whose potential drop is $\phi_{s}$. The thermionic electric current is modelled according to the RichardsonDushman equation (27) and the Schottky effect [20].

$$
J=A T^{2} \exp \left(-e \phi_{w f} / k_{B} T\right)
$$

where $A$ is the Richardson constant with a value of $120 \mathrm{~A} /\left(\mathrm{cm}^{2} \mathrm{~K}^{2}\right), T$ is the insert temperature, $k_{B}$ is the Boltzmann constant, $e$ is the elementary charge and $\phi_{w f}$ is the work function of the insert material. The Schottky effect is a 


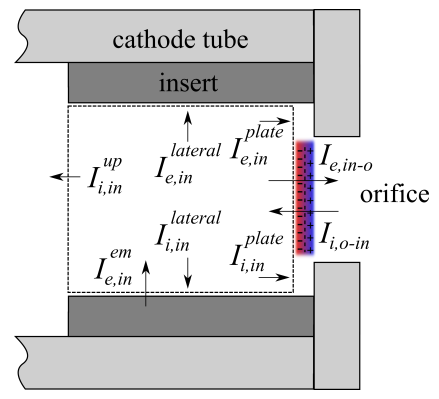

Figure 3: Current continuity in the insert region.

phenomenon in which the thermionic emission is enhanced due to the presence of an external electric field that reduces the amount of energy required by the electron to escape the insert. In other words, the work function of the material is reduced and the effective work function is [20]

$$
\phi_{e f f}=\phi_{w f}-\sqrt{\frac{e E_{c}}{4 \pi \epsilon_{0}}},
$$

where $\phi_{w f}$ is the work function. For a $\mathrm{BaO}$ insert, the work function is [31]

$$
\phi_{w f}=1.67+2.82 \cdot 10^{-4} T_{w, \text { in }} \quad[\mathrm{V}] .
$$

The electric field $E_{c}$ in equation (28) is the electric fields at the cathode sheath $[16,32]$

$$
E_{c}=\sqrt{\frac{n_{e, i n} e T_{e, i n}}{\epsilon_{0}}}\left[2 \sqrt{1+2 \frac{\phi_{s}}{T_{e, i n}}}-4\right]^{1 / 2},
$$

where the $T_{e, i n}$ is in $\mathrm{eV}$. Note that $E_{c}$ depends on the potential drop across the sheath $\phi_{s}$. Finally, the equation of the field-enhanced thermionic electric current is

$$
I_{e, i n}^{e m}=D T_{w, i n}^{2} \exp \left(-e \phi_{e f f} / k_{B} T_{w, i n}\right) A_{i n, l a t},
$$

where $D=120 \cdot 10^{4} \mathrm{~A} / \mathrm{m}^{2} \mathrm{~K}^{2}$ is the Richardson constant of the $\mathrm{BaO}$ insert, $T_{w, \text { in }}$ is the insert temperature, and $A_{i n, l a t}=2 \pi r_{i n} L_{i n}$ is the insert lateral surface.

The following sections cover the equation of conservation regarding the insert region.

\subsubsection{Current continuity}

As in the orifice case, the current continuity equation requires that the charge within the insert volume does not change. Following the schematic in Figure 3, the electron and ion currents are balanced as follows:

$$
-I_{i, i n}^{u p}-I_{e, i n}^{e m}-I_{i, i n}^{\text {lateral }}+I_{e, i n}^{\text {lateral }}-I_{i, \text { in }}^{\text {plate }}+I_{e, \text { in }}^{\text {plate }}+I_{e, \text { in }-o}+I_{i, i n-o}=0,
$$


where

$$
I_{i, i n}^{u p}=\frac{e}{4} n_{e, i n} \sqrt{\frac{8 e T_{i, i n}}{\pi m_{\mathrm{Xe}}}} A_{i n}
$$

is the random ion current flowing upstream, $A_{i n}=\pi r_{i n}^{2}$ is the insert region cross section, $n_{e, i n}$ is the plasma density in the insert region;

$$
I_{i, \text { in }}^{\text {lateral }}=e 0.606 n_{e, \text { in }} \sqrt{\frac{e T_{e, i n}}{m_{\mathrm{Xe}}}} A_{\text {in }, l a t}
$$

is the ion current on the lateral surface of the insert (assumed a presheath and the validity of the Bohm condition);

$$
I_{e, \text { in }}^{\text {lateral }}=\frac{e}{4} n_{e, i n} \sqrt{\frac{8 e T_{e, \text { in }}}{\pi m_{e}}} \exp \left(-\frac{\phi_{s}}{T_{e, \text { in }}}\right) A_{\text {in }, \text { lat }}
$$

is the random electron current on the lateral insert surface assumed a Maxwellian distribution at an average electron velocity $\bar{v}_{e, i n}=\sqrt{8 e T_{e, i n} / \pi m_{e}}$; currents $I_{i, \text { in }}^{\text {plate }}$ and $I_{e, \text { in }}^{\text {plate }}$ are described as in equations (34) and (35), respectively, using the plate surface $A_{\text {plate }}=\pi\left(r_{i n}^{2}-r_{o}^{2}\right)$. Finally, currents $I_{i, i n-o}$ and $I_{e, i n-o}$ are previously shown in equations (12) and (13), respectively.

The potential drop $\phi_{s}$ is one of the unknown plasma properties and it can be calculated by solving the following implicit equation, which is derived by combining equations (9), (14) and (32):

$I_{D}=I_{i, i n}^{u p}+I_{e, i n}^{e m}\left(\phi_{s}\right)+I_{i, i n}^{\text {lateral }}-I_{e, \text { in }}^{\text {lateral }}\left(\phi_{s}\right)+I_{i, i n}^{\text {plate }}-I_{e, \text { in }}^{\text {plate }}\left(\phi_{s}\right)+I_{i, o}^{\text {lateral }}-I_{e, o}^{\text {lateral }}\left(\phi_{s}\right)$.

\subsubsection{Ion mass conservation}

Turning now to the conservation of ion mass, the ions are produced within the insert by ionisation and transported into the insert from the orifice region through the double sheath. At the same time, ions are lost through the sheaths of the insert and the plate surfaces and upstream due to thermal ion motion:

$$
\begin{aligned}
\frac{d N_{i, i n}}{d t} & =0= \\
& =\underbrace{V_{i n}\left(n_{n, i n}-n_{e, i n}\right.}) n_{e, i n} K_{i z, \text { in ionisation }}+ \\
& +\underbrace{0.606 n_{e, o} v_{B, o} A_{o}}_{\text {ions from orifice }}+ \\
& -\underbrace{0.606 n_{e, i n} v_{B, i n}\left(A_{i n}+A_{\text {in }, \text { lat }}\right)}_{\text {upstream }}+ \\
& -\underbrace{\frac{n_{e, \text { in }}}{4} \bar{v}_{i, \text { in }} A_{i n}}_{\text {lateral walls and plate sheath }} .
\end{aligned}
$$


The first, third and fourth terms can be commented similarly as it is done in the orifice section C.2.3.2, with the main difference that, in this cases, the subscript " $i n$ " refers to the values in the insert region, e.g. $T_{e, i n}$ is used in $v_{B, i n}$. The second term takes into account the ions transported from the orifice region through the double sheath. Equation (37) is useful to derive the electron temperature $T_{e, i n}$ in the insert region.

\subsubsection{Power balance}

The last equation that is part of the system of the equations of conservation is the power balance within the insert volume. Three factors contribute to the plasma heating, namely the Joule heating, the energy per unit time carried by the thermionically emitted electrons and the energy per unit time transported by the orifice ions that cross the double sheath. The power losses are due to ionisation collisions, ion and electron energy transport toward the sheaths (lateral walls and orifice plate), the energy transported by the thermal ions that flow upstream and that transported by electron convection. The power balance equation is

$$
\begin{aligned}
& \underbrace{R_{\Omega, i n}\left(I_{i, o-i n}+I_{e, i n-o}\right)^{2}}_{\text {Joule heating }}+ \\
& +\underbrace{I_{i, o-i n}\left(\frac{T_{e, o}}{2}+V_{d s}\right)}+ \\
& \text { presheath }+ \text { double sheath } \\
& +\underbrace{I_{e, i n}^{e m}\left(2 \frac{k_{B}}{e} T_{w, i n}+\phi_{s}\right)}_{\text {thermionic electrons }}= \\
& =\underbrace{\left(I_{i, \text { in }}^{u p}+I_{i, \text { in }}^{\text {lateral }}+I_{i, \text { in }}^{\text {plate }}\right) E_{i z}}_{\text {ionisation }}+ \\
& +\underbrace{\left(I_{i, \text { in }}^{\text {lateral }}+I_{i, \text { in }}^{\text {plate }}\right)\left(\frac{T_{e, \text { in }}}{2}+\phi_{s}\right)}_{\text {ions on lateral wall and orifice plate }}+ \\
& +\underbrace{I_{i, i n}^{u p}\left(2 T_{i, i n}\right)}_{\text {ions upstream }}+ \\
& +\underbrace{\left(I_{e, \text { in }}^{\text {lateral }}+I_{e, \text { in }}^{\text {plate }}\right)\left(2 T_{e, i n}\right)}_{\text {electrons on lateral wall and orifice plate }}+ \\
& +\underbrace{I_{e, i n-o} \frac{5}{2} T_{e, i n}}_{\text {electron convection }} \text {. }
\end{aligned}
$$


The first term on the left hand side of equation (38) is the Joule heating, where the plasma resistance is

$$
R_{\Omega, i n}=\eta \frac{r_{i n}}{4 / 3 \pi r_{i n} L_{i n}} .
$$

The plasma resistivity $\eta$ in the insert volume is calculated similarly to that in the orifice section from equations (21), (22), (23), (24) and (25) in which the plasma and neutral gas properties must be those relative to the insert region. Within the insert volume, it is assumed that the main current flow is along the radial direction, for this reason the plasma resistivity is multiplied by the fraction $r_{i n} /\left(4 / 3 \pi r_{i n} L_{i n}\right)$, which is the path along which the plasma flows $\left(r_{i n}\right)$ divided by the averaged cross section perpendicular to the radial direction, which is computed as $[11,19]$

$$
\begin{aligned}
\left\langle A_{\text {in }, \text { lat }}\right\rangle_{S} & = \\
& =\frac{\int_{S} A_{i n, l a t}(r) d S}{\int_{S} d S}= \\
& =\frac{\int_{0}^{2 \pi} \int_{0}^{r_{i n}} A_{\text {in }, l a t}(r) r d r d \theta}{\int_{0}^{2 \pi} \int_{0}^{r_{i n}} r d r d \theta}= \\
& =\frac{2 \pi \int_{0}^{r_{i n}} 2 \pi r L_{i n} r d r}{2 \pi \int_{0}^{r_{i n}} r d r}= \\
& =\frac{4}{3} \pi r_{i n} L_{i n} .
\end{aligned}
$$

The current that contributes to the Joule heating is approximated by the total current flowing from the insert to the orifice, i.e. $\left(I_{i, o-i n}+I_{e, i n-o}\right)$.

The second term on the left hand side of equation (38) is the contribution of the ions born in the orifice region that pass through the double sheath. They carry an energy equal to $\left(0.5 T_{e, o}+V_{d s}\right)$ due to the acceleration across the presheath in the orifice region and the double sheath.

The last term on the left hand side of equation (38) takes into account the energy per unit time carried by the thermionically emitted electrons. Their total energy is the sum of the average kinetic energy the electrons gain in the insert, in which it is assumed a Maxwellian distribution of the electron population within the insert material, and the acceleration experienced across the sheath.

The first term on the right hand side of the power balance equation is the ionisation loss, where $E_{i z}$ is the ionisation energy. Note that the ionisation loss is in the form of surface loss rather than in the form of volumetric loss as in equation (19).

The second term on the right hand side of the power balance equation is the sum of the power losses due to the ions leaving the plasma through the sheaths of the lateral and orifice plate surfaces. Their total energy is the sum of the energy at the presheath and voltage drop across the sheath.

The third term on the right hand side of equation (38) takes into account the 
power lost due to the ions leaving the plasma upstream. Their average kinetic energy is $2 T_{i, i n}$ assuming a Maxwellian distribution.

The fourth term on the right hand side of equation (38) is the sum of the power losses due to the electron random flux toward the lateral wall and the orifice plate.

The last term is the power transported by the electrons due to convection toward the orifice region.

The power balance in equation (38) is numerically solved with respect to the electron density $n_{e, i n}$. In the following section, the solution procedure is explained.

\subsection{Algorithm}

The solution procedure is as follows. First, the geometrical dimensions of the orifice and insert are selected by the user, i.e. $r_{o}, L_{o}, r_{i n}$ and $L_{i n}$. Then, the operating conditions of the cathode are set, i.e. the discharge current $I_{D}$ and the xenon mass flow rate $\dot{m}_{\mathrm{Xe}}$. The temperatures of the orifice and insert walls are set according to experimental data or by solving the Richardson-Dushman equation (31) for $I_{e, i n}^{e m} \approx I_{D}$. The code requires the initial guesses of the sheath potential $\phi_{s}$ (a value of $8 \mathrm{~V}$ is used [13]), electron density and temperature in the insert region (these are set equal to $n_{e, \text { in }}=10^{21} \mathrm{~m}^{-3}$ and $T_{e, \text { in }}=1 \mathrm{eV}$ ). After the inputs from the user are collected, the code solves equations (1) and (5) in order to calculate the neutral density within the orifice and the insert volumes. The plasma model has two loops, an external loop and an internal loop. The first iteration of the external loop starts by simultaneously solving the equations of conservation (17) and (19) in the orifice region with respect to the unknown electron density $n_{e, o}$ and electron temperature $T_{e, o}$. The internal loop is required to iteratively solve equations (36), (37) and (38) with respect to $\phi_{s}$, $T_{e, \text { in }}$ and $n_{e, i n}$, respectively. The solutions converge when the variation of the solutions of the electron density $n_{e, i n}$ and electron temperature $T_{e, i n}$ between two successive iterations is below $0.01 \%$. After the internal loop ends, the values of the calculated $\phi_{s}, T_{e, \text { in }}$ and $n_{e, \text { in }}$ are used as updated inputs in the orifice region. Thus, the second iteration of the external loop starts. The plasma model ends when the variation of the electron densities in the insert and orifice regions between two consecutive external loops is below the threshold $0.01 \%$. The flowchart of the algorithm is shown in Figure 4. The convergence on a unique result has been tested. This has been done by studying the dependence of the terms in the equations of conservation on the unknown parameters. It has been found that, within the typical ranges explored, the right-hand side and the left-hand side of these equations are monotonic functions of the unknown variables. The intersection of two monotonic equations is a point, hence, a unique solution. The algorithm stops only if convergence on a unique solution is reached. If it does not converge, the plasma solver continues until a large number of iterations - set by the user - is reached. Typically, the algorithm converges within 100 iterations 


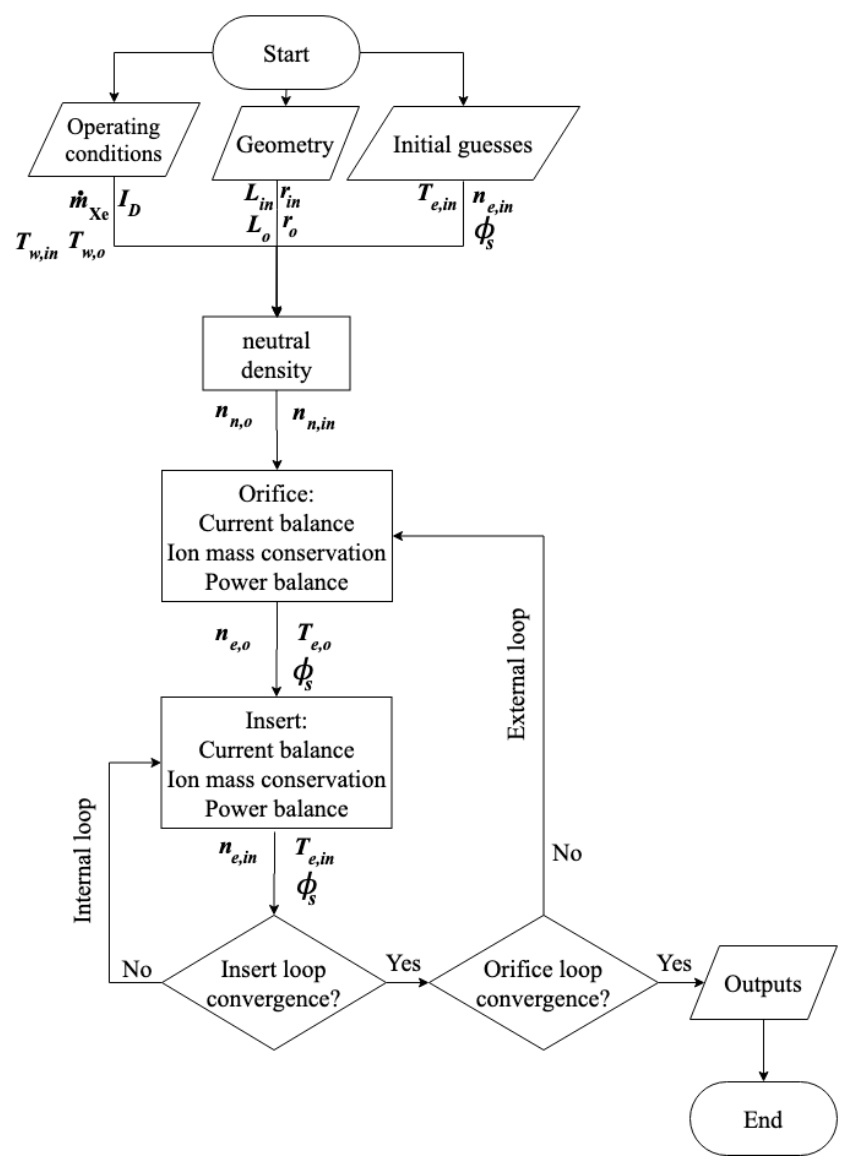

Figure 4: Flowchart of the plasma model algorithm.

\subsection{Operational parameters}

The main macroscopic parameters that are monitored during a hollow cathode operation are the discharge power, current and voltage. The discharge power is computed as

$$
P_{D}=I_{e, i n}^{e m} \phi_{s}+R_{\Omega, i n}\left(I_{i, o-i n}+I_{e, i n-o}\right)^{2}+\left(I_{i, o-i n}+I_{e, i n-o}\right) V_{d s}+R_{\Omega, o} I_{D}^{2},
$$

where $P_{s}=I_{e, i n}^{e m} \phi_{s}$ is the power spent to accelerate the thermionically emitted electrons through the sheath; $P_{\Omega, i n}=R_{\Omega, \text { in }}\left(I_{i, o-i n}+I_{e, i n-o}\right)^{2}$ is the Joule heating in the insert region; $P_{d s}=\left(I_{I, o-i n}+I_{e, i n-o}\right) V_{d s}$ is the power spent to accelerate ions and electrons across the double sheath; and $P_{\Omega, o}=R_{\Omega, o} I_{D}^{2}$ is the Joule heating in the orifice region. Note that the discharge power is slightly 
Table 1: Geometrical dimensions and operating conditions of a sample hollow cathode neutraliser.

\begin{tabular}{l|c}
\hline \hline \multicolumn{1}{c}{ Parameter } & Value \\
\hline Orifice diameter $2 r_{o}[\mathrm{~mm}]$ & 0.3 \\
Orifice length $L_{o}[\mathrm{~mm}]$ & 0.8 \\
Insert diameter $2 r_{i n}[\mathrm{~mm}]$ & 1.0 \\
Insert length $L_{i n}[\mathrm{~mm}]$ & 10.0 \\
Insert temperature $T_{w, i n}[\mathrm{~K}]$ & 1310 \\
Insert material $(\mathrm{BaO})$ work function $\phi_{w f}[\mathrm{~V}]$ & $1.67+2.82 \cdot 10^{-4} T_{w, i n}$ \\
Discharge current $I_{D}[\mathrm{~A}]$ & 1.0 \\
Xenon mass flow rate $\dot{m}_{\text {Xe }}[\mathrm{mg} / \mathrm{s}]$ & 0.039 \\
\hline \hline
\end{tabular}

lower than the total input power in the simulated regions, which is

$P_{t o t}=I_{e, i n}^{e m}\left(2 \frac{k_{B}}{e} T_{w, i n}+\phi_{s}\right)+R_{\Omega, i n}\left(I_{i, o-i n}+I_{e, i n-o}\right)^{2}+\left(I_{i, o-i n}+I_{e, i n-o}\right) V_{d s}+R_{\Omega, o} I_{D}^{2}$.

The power difference is the power introduced in the insert volume by the electrons having a not-zero initial kinetic energy (before passing through the sheath) due to the hot insert, i.e.

$$
P_{t o t}-P_{D}=I_{e, i n}^{e m} 2 \frac{k_{B}}{e} T_{w, i n}
$$

which is about $0.1-0.2 I_{e, i n}^{e m}[\mathrm{~W}]$. The discharge current is $I_{D}$ and the discharge voltage is calculated as

$$
V_{D}=\frac{P_{D}}{I_{D}}
$$

It is possible to estimate the power deposited on the internal surfaces of the cathode due to ion and electron bombardment. These power terms can be used as inputs in a thermal model to estimate the orifice and insert wall temperatures. The model here presented simulates the insert and orifice regions, excluding the cathode-to-keeper region and the keeper sheath. Therefore, the total power computed in this model does not include the power in the cathode-to-keeper region.

\section{Results and discussions}

The results of the plasma model are here discussed for a sample cathode, whose geometrical dimensions and operating conditions are listed in Table 1. In order to investigate the behaviour of the model, the results have been studied at various discharge and geometrical conditions. It is true that the discharge current and the insert temperature are strictly related quantities [33], however, since 


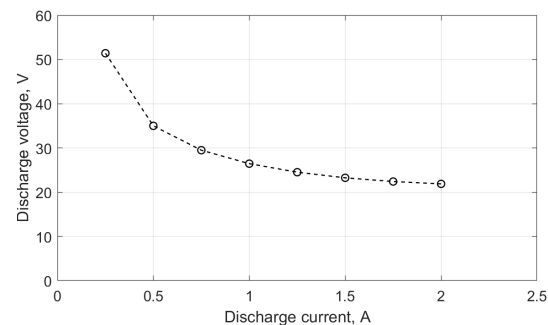

(a)

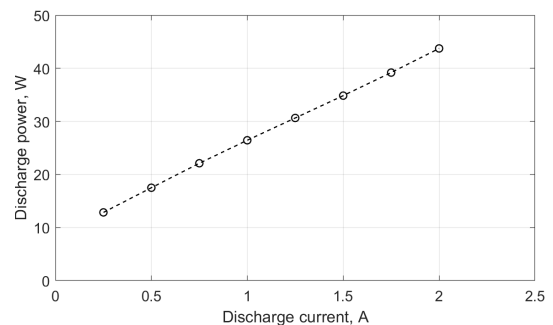

(b)

Figure 5: (a) $I-V$ characteristic and (b) discharge power versus discharge current of the simulated HCN. Geometry and discharge condition are shown in Table 1.

this plasma model is not yet coupled with a thermal model, the main focus is to examine the qualitative trend of the results.

\subsection{Effect of the discharge current}

The simulated $I$ - $V$ characteristic and the discharge power as functions of the discharge current of the hollow cathode neutraliser $(\mathrm{HCN})$ are shown in Figure 5. Both trends are consistent with the experimental data of other hollow cathodes [34, 35]. The distribution of power among $P_{s}$, $P_{\Omega, i n}, P_{d s}$ and $P_{\Omega, o}$ versus the discharge current is shown in Figure 6 . According to the model, the fraction of Joule heating in the orifice region increases while the fraction of power in the insert region decreases as the discharge current is increased. In absolute values, all powers increase

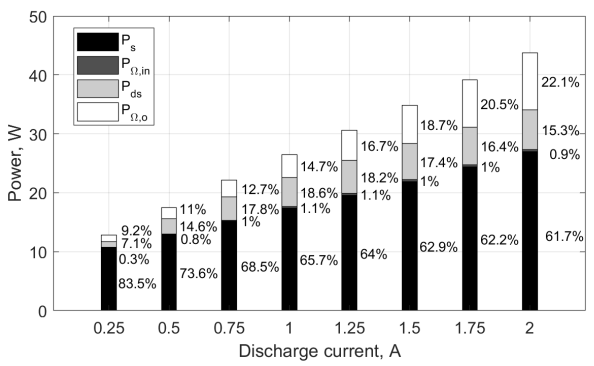

Figure 6: Power distribution as function of the discharge current. Geometrical and discharge condition are shown in Table 1. with the increased current. These behaviours have also been predicted by Albertoni's model [16].

In Figure 7, the experimental $I-V$ characteristic of a $\mathrm{LaB}_{6}$ cathode [33] is compared with the simulation outputs of our model, where the insert work function chosen is $\left(2.66+1.23 \cdot 10^{-4} T_{w, i n}\right) \mathrm{V}$ [13]. The cathode consists of a $\mathrm{LaB}_{6}$ emitter of $3 \mathrm{~mm}$ in inner diameter and $6 \mathrm{~mm}$ in axial length and an orifice of $0.4 \mathrm{~mm}$ in diameter and long $0.36 \mathrm{~mm}$. Given an assumed insert temperature varying from $1750 \mathrm{~K}$ to $1790 \mathrm{~K}$ as the discharge current increases, our plasma model predicts the discharge voltage trend within an $8 \mathrm{~V}$ interval from the experimental points. 


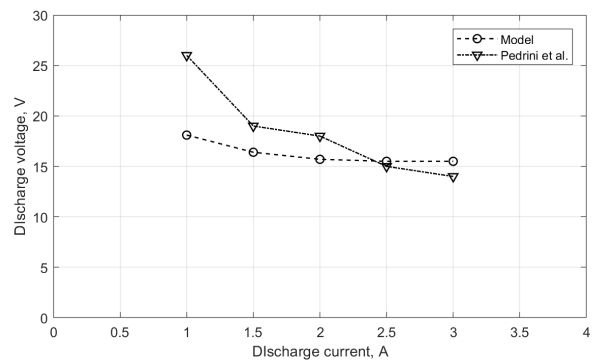

Figure 7: Comparison of experimental and simulated $I-V$ characteristics of a $\mathrm{LaB}_{6}$ hollow cathode. Experimental data from Pedrini et al. [33].

Note that the experimental discharge voltage is the cathode-to-keeper voltage, which includes the voltage drop across the upstream the cathode orifice and the keeper sheath voltage drop. Our model estimates the voltage drop upstream the cathode orifice, excluding the contribution of the cathode-to-keeper region.

\subsection{Effect of the orifice geometry}

Let us consider now the model outputs when the controlled parameter is the orifice diameter. As shown in Figure 8a, the electron temperature in the insert and orifice volumes increases as the orifice diameter is enlarged. This is strictly related to the plasma density profile shown in Figure 8b. The plasma density in both modelled regions decreases with increasing orifice diameter and, as a consequence, the number of collisions reduces, yielding a higher average electron temperature at larger orifice diameters. The lower plasma density is explained by a decrease of the number of ionising collisions, since the neutral density diminishes as the orifice is enlarged. Figure $8 \mathrm{c}$ is the bar plot of the power fractions as functions of the orifice diameter size. It can be noticed that the Joule heating in the orifice sharply increases at small orifice diameters. This is due to the relation $P_{\Omega, o} \sim 1 /\left(2 r_{o}\right)^{2}$ at very small orifice diameters and fixed discharge current. As the orifice diameter decreases, the power spent in the double sheath acceleration increases both in fraction and absolute value. This is explained by the dependence of the power across the double sheath to the electron temperature, electron density and orifice cross section as shown in equation (8)

The plasma properties respond differently when the orifice length, instead of the orifice diameter, is the controlled parameter. The model predicts that the electron temperature slightly decreases in both the orifice and insert volumes as the orifice length increases, as shown in Figure 9a. At the same conditions, the electron density slightly rises with increasing orifice length, as shown in Figure $9 \mathrm{~b}$. Finally, the bar plot of the power fractions in Figure 9c shows that the main contribution to the discharge power comes from the Joule heating in the orifice $P_{\Omega, o}$. While there is not a substantial change in the power fractions of $P_{\Omega, i n}$ 


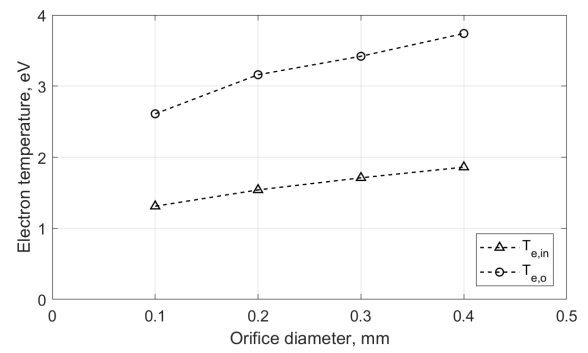

(a)

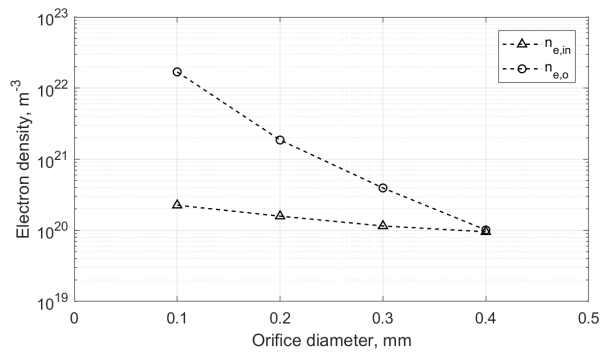

(b)

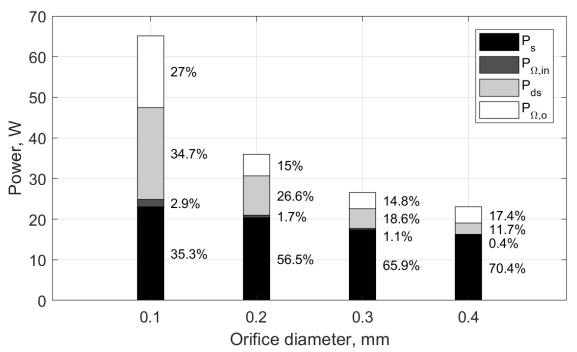

(c)

Figure 8: Electron temperature, electron density and power fraction distribution as function of the orifice diameter. 
and $P_{d s}$, the power at the cathode sheath increases, mainly due to an increase of the sheath potential $\phi_{s}$ with $L_{o}$. The latter is in contrast with the prediction of Albertoni's model, mainly due to the different approach to estimate $\phi_{s}$ and the absence of a plasma-thermal coupling in our model.

\subsection{Effect of the insert geometry}

The response of the modelled plasma properties and power inputs with respect to changes in the insert geometries are shown in Figures 10 and 11. Generally, if either the insert radius or the insert length is increased the electron temperature does not change in the orifice region, and it slightly decreases in the insert region. The electron density is expected to slightly increase in the orifice volume and to decrease in the insert region. As expected, the discharge power rises as the insert diameter becomes larger. As seen in Figures 10c and 11c, both $P_{s}$ and $P_{d s}$ increase in absolute value and in fraction, whereas the contribution of $P_{\Omega, o}$ reduces.

\subsection{Effect of the mass flow rate}

Let us consider the effect that the increase of the mass flow rate has on the electron temperature, electron density and power consumption. As shown in Figure 12a, the orifice electron temperature decreases as the xenon mass flow rate rises from $0.029 \mathrm{mg} / \mathrm{s}(0.3 \mathrm{sccm})$ to $0.098 \mathrm{mg} / \mathrm{s}(1 \mathrm{sccm})$. A less steep slope is observed for the electron temperature in the insert region. In Figure $12 \mathrm{~b}$, it is seen that the modelled electron density in the orifice region increases at a higher rate than that of the electron density in the insert region, the latter being almost constant across the mass flow rate investigated. These $T_{e}$ and $n_{e}$ profiles can be explained by an increase of the collisional events, especially in the orifice region, as the neutral pressure rises. The bar plot in Figure 12c shows the discharge power and the fractional contribution of the four power terms listed in equation (41) as functions of the xenon mass flow rate. The model predicts that, while the power consumption due to Joule heating increases, the overall discharge power slightly drops as the mass flow rate increases.

\subsection{Power density deposition}

The power density, in $\mathrm{W} / \mathrm{mm}^{2}$, deposited on the internal surfaces of the cathode due to ion and electron bombardment is shown in Figure 13. Figures 13a, $13 \mathrm{~b}$ and $13 \mathrm{c}$ illustrate the power density deposited on the orifice lateral surface, orifice plate and insert, respectively, as a function of the orifice diameter. Similarly, Figures 13d, 13e and 13f show the power densities as functions of the insert diameter. In general, the orifice walls are subject to a higher power 


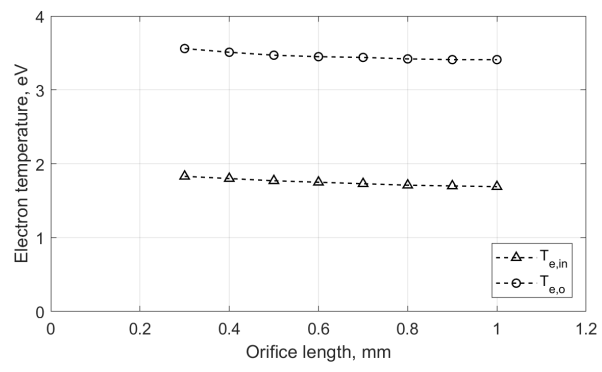

(a)

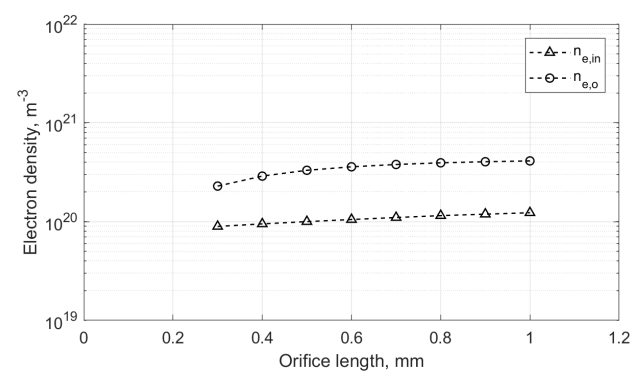

(b)

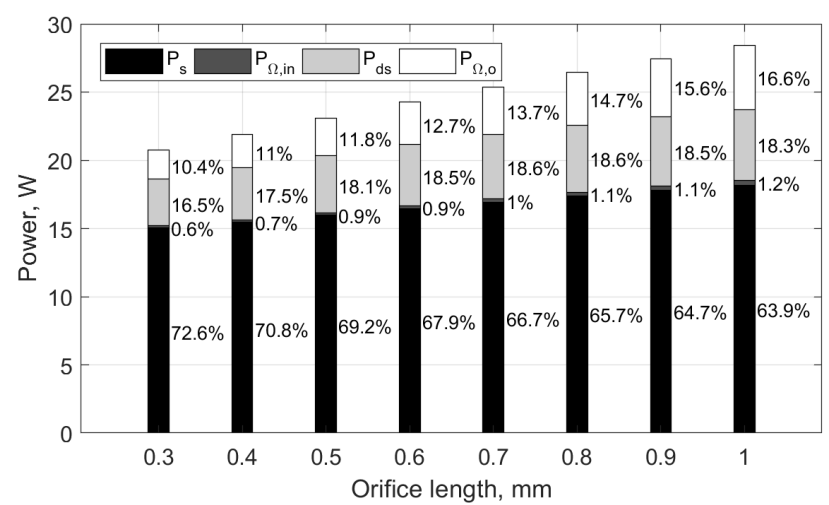

(c)

Figure 9: Electron temperature, electron density and power fraction distribution as function of the orifice length. 


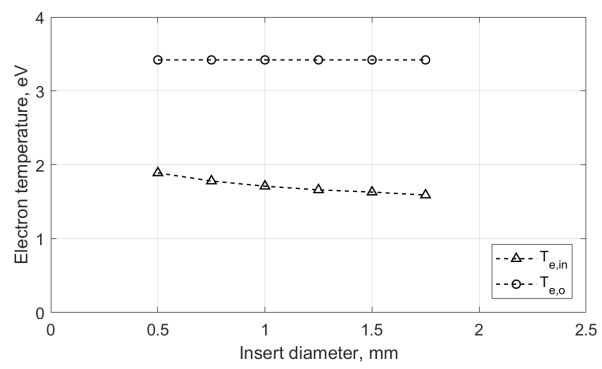

(a)

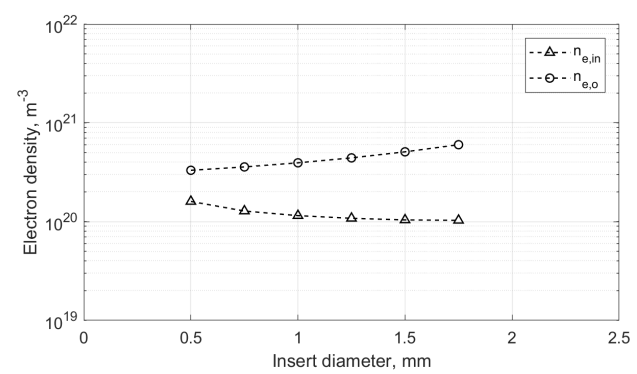

(b)

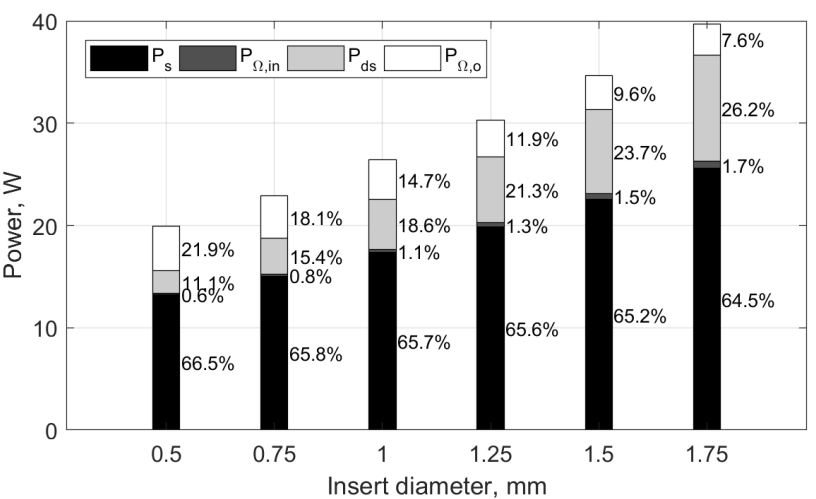

(c)

Figure 10: Electron temperature, electron density and power fraction distribution as function of the insert diameter. 


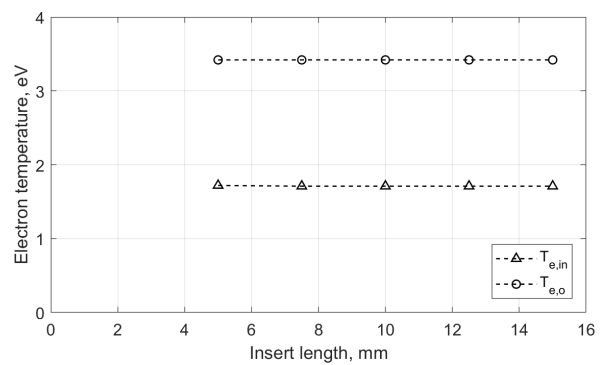

(a)

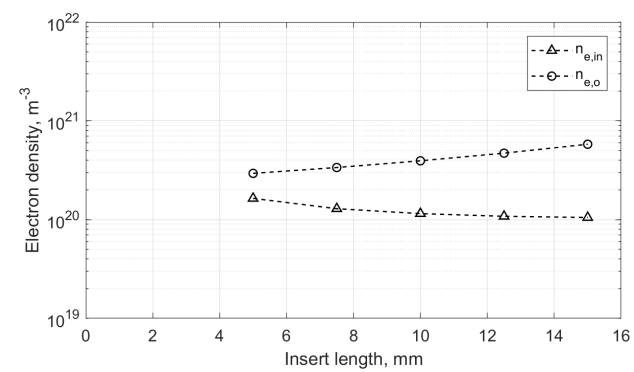

(b)

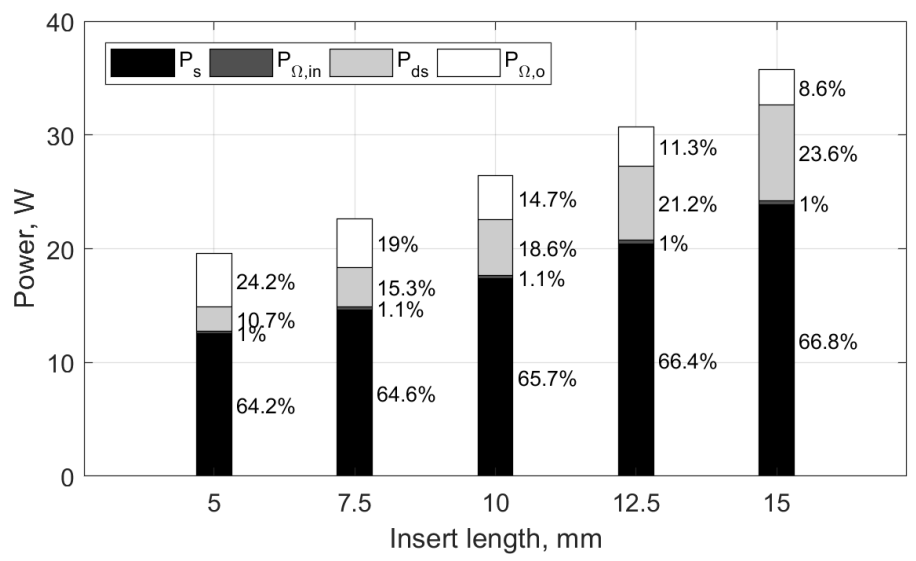

(c)

Figure 11: Electron temperature, electron density and power fraction distribution as function of the insert length. 


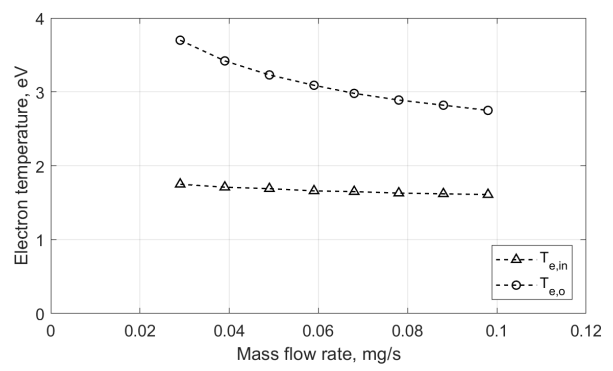

(a)

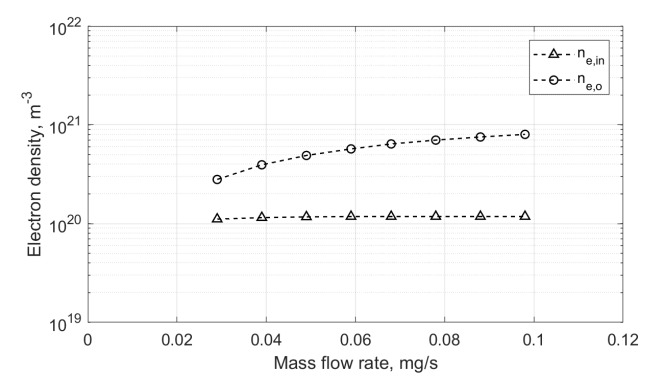

(b)

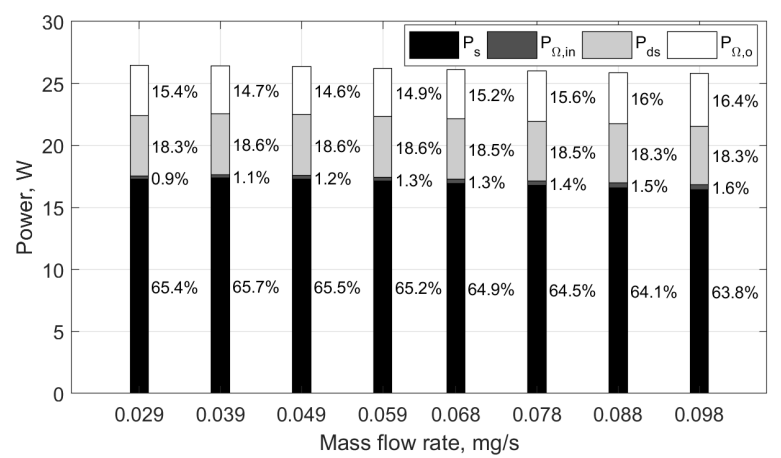

(c)

Figure 12: Electron temperature, electron density and power fraction distribution as function of the xenon mass flow rate. 


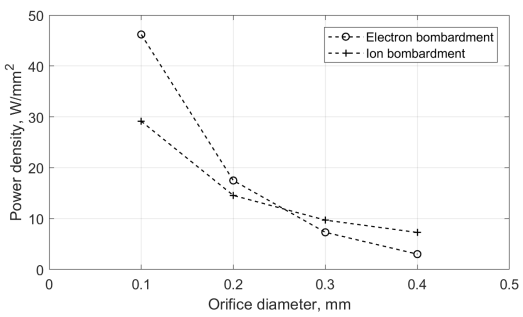

(a) Orifice lateral surface.

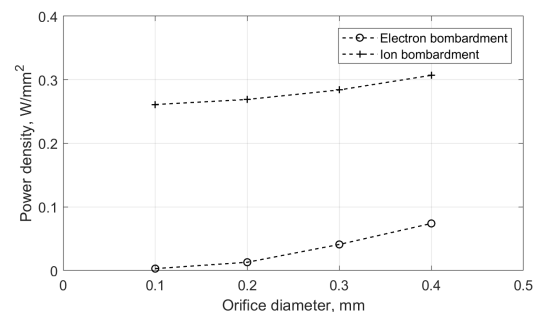

(b) Orifice plate surface.

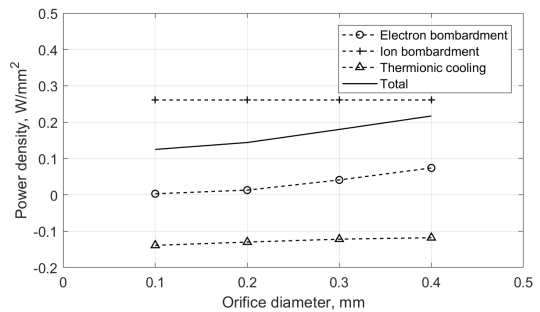

(c) Insert surface.

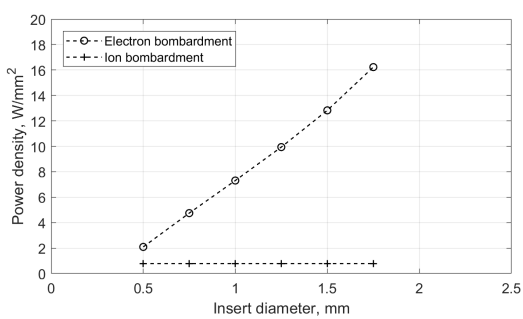

(d) Orifice lateral surface.

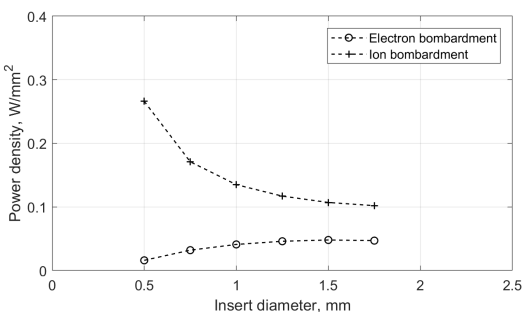

(e) Orifice plate surface.

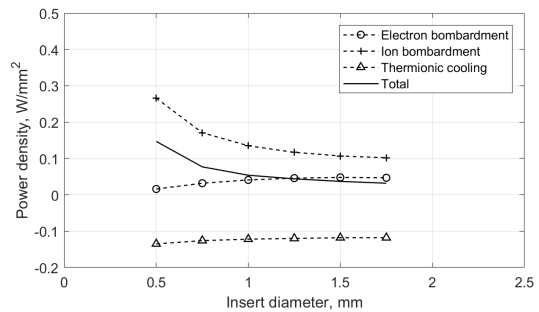

(f) Insert surface.

Figure 13: Power densities on (a) (d) orifice lateral surface, (b) (e) orifice plate surface and (c) (f) insert lateral surface as functions of the orifice and insert diameters. 
Table 2: Power consumption and power deposition in the modelled cathode, whose geometry and discharge condition

are listed in Table 1.

\begin{tabular}{l|c}
\multicolumn{1}{c}{ Parameter } & {$[\mathrm{W}]$} \\
\hline Total input power $P_{t o t}$ & 26.8 \\
Discharge power $P_{D}$ & 26.2 \\
Power transported by extracted electrons and ions & 8.6 \\
Power re-deposited into the cathode & 18.2 \\
\hline Electron bombardment on orifice lateral surface & 5.5 \\
Ion bombardment on orifice lateral surface & 0.5 \\
Electron bombardment on orifice plate & $\approx 0.0$ \\
Ion bombardment on orifice plate & 0.1 \\
Electron bombardment on insert lateral surface & 1.3 \\
Ion bombardment on insert lateral surface & 4.2 \\
Power associated with upstream ion flow, & 6.6 \\
excitation and ionisation events & \\
\hline \hline
\end{tabular}

density $\left(5-50 \mathrm{~W} / \mathrm{mm}^{2}\right)$ compared to that deposited on the other internal surfaces of the cathode $\left(0-0.3 \mathrm{~W} / \mathrm{mm}^{2}\right)$. The high power density deposited on the orifice lateral surface implies that the orifice is prone to erosion, in particular at small diameters. Figures 13a and 13d show the electron and ion power density deposition as the orifice diameter and the insert diameter are increased, respectively. The electron bombardment is the main contributor to the power density deposited on the orifice lateral wall, in both cases. As the orifice diameter is increased, the power density due to electrons decreases. On the other hand, as the insert diameter is increased the power density due to electrons increases. The latter is explained by the fact that as the thermionic surface increases, more electrons flow into the insert plasma and, due to the current conservation in equation (36), the sheath potential needs to decrease to keep the currents balanced. Since $\phi_{s}$ is lowered, more electrons can reach the orifice wall. As shown in Figures 13b and 13e, the heating of the orifice plate surface is mainly due to ion bombardment. Figures $13 \mathrm{c}$ and $13 \mathrm{f}$ show the power densities due to the electron and ion bombardment, the cooling of the insert due to thermionically emitted electrons and the total power density deposited on the insert. The total power density is the sum of the ion and electron contributions less the cooling due to the thermionically emitted electrons. These results show that the heating mechanism of cathodes whose insert and orifice radii are almost equal is normally ion bombardment of the insert, as predicted by previous studies (Ref. [13] pp.248-249).

\subsection{Power budget}

The power consumption, total input power and power deposition regarding 


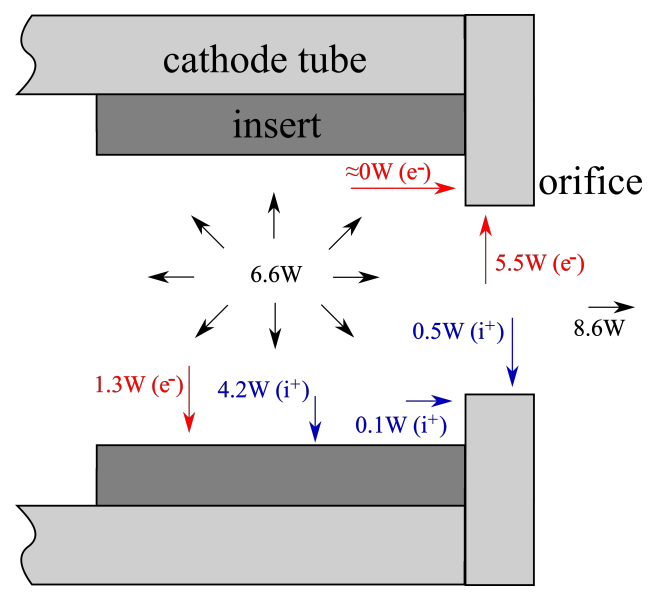

Figure 14: Visualisation of power deposition and transport in the modelled cathode.

the sample cathode (see Table 1) are shown in Table 2 and visualised in Figure 14. Among the power listed there is that one transported by electrons and ions extracted through the cathode orifice, the ion and electron bombardment on various internal surfaces and the amount of power that stays trapped into the cathode. The last row of Table 2 is the sum of the power due to ions flowing upstream the insert, excitation collisions and ionisation collisions. The energy associated to the last two terms is mainly redistributed on the cathode internal surfaces, for example via radiative light emission subsequent to a collisional excitation event.

\section{Conclusions}

The zero-dimensional model here presented introduces the following revisions to previously published models: (i) a revised design of the physical processes, e.g. the effects of a same discharge event are included in all the equations of conservation, (ii) inclusion of terms neglected in previous models and (iii) calculation of ion and electron bombardment power deposited on the surfaces of the insert, orifice plate and orifice. The quantitative response of the model to variation of geometrical and operational condition of a sample cathode are presented. The numerical results are in accordance with experimental results published in literature.

This model can be used to design both conventional HCNs (e.g. $\mathrm{LaB}_{6}$ and $\mathrm{BaO}$ cathodes) and $\mathrm{HCNs}$ whose thermionic material is not affected by poisoning. Estimations of the discharge power, power distribution among the plasma regions and power deposition onto the cathode walls are provided. There are 
some limitations of the model here presented and the following is suggested to increase its self-consistency. It is recommended to couple a thermal solver that accepts the power deposited on the internal walls of the cathode as an input. In this way, the temperature of the insert and orifice walls is directly derived in a unified plasma-thermal solver. With regard to the plasma model, the following improvements are suggested: (i) the neutral pressure in the insert region should be described by a transitional flow law instead as a Poiseuille flow, since the Knudsen number in the insert region is $0.01<\mathrm{Kn}<1$; (ii) the assumed neutral and ion temperatures should take into account heating processes that cause these temperature to be two to four times that of the cathode walls; (iii) the derived neutral density should take into account the neutral loss mechanism due to ionisation event; (iv) the contribution of the current due to thermionic emission from the orifice surface should be included into the current continuity equation - this is especially relevant for simulating thermionic cathodes whose orifice is made by low-work-function materials [36]; and (v) inclusion of stepwise ionisation as ionisation pathway by coupling an existing xenon collisional-radiative

model [37] with the hollow cathode model. The assumptions at the base of the model are in any case well-established in the background literature and the resulting uncertainty tested. The model found an immediate exploitation in the sizing of HCNs at the Surrey Space Centre and its industry partners.

\section{Acknowledgments}

The activities were performed in the frame of the project "Development of a Halo-200 Electric Propulsion System" supported by the UK Space Agency.

\section{References}

[1] M. Coletti, R. I. Marques, S. B. Gabriel, Discharge Hollow Cathode Design for a 4-Gridded Ion Engine, in: 2010 IEEE Aerospace Conference, Vol. 6-13 March, Big Sky, Montana, USA, 2010. doi:10.1177/0954410011409131.

[2] J. D. Sommerville, L. B. King, Effect of Cathode Position on HallEffect Thruster Performance and Cathode Coupling Voltage, in: 43rd AIAA/ASME/SAE/ASEE Joint Propulson Conference \& Exhibit, no. 8-11 July, Cincinnati, Ohio, USA, 2007, pp. AIAA-2007-5174. doi:10.2514/ $6.2007-5174$.

[3] A. Lucca Fabris, A. Knoll, K. Dannenmayer, T. Schönherr, T. Potterton, P. Bianco, Vacuum Facility Effects on Quad Confinement Thruster Testing, 35th International Electric Propulsion Conference (8-12 October) (2017) IEPC-2017-250.

[4] D. E. Siegfried, P. J. Wilbur, An investigation of mercury hollow cathode phenomena, in: 13th International Electric Propulsion Conference, no. 2527 April, San Diego, California, USA, 1978, pp. IEPC-78-705. doi:10. 
$2514 / 6.1978-705$.

URL http://arc.aiaa.org/doi/10.2514/6.1978-705

[5] D. E. Siegfried, P. J. Wilbur, Phenomenological model describing orificed, hollow cathode operation, AIAA Journal 21 (1) (1983) 5-6. doi:10.2514/ 3.8022 .

URL http://arc.aiaa.org/doi/10.2514/3.8022

[6] D. E. Siegfried, P. J. Wilbur, A model for mercury orificed hollow cathodes: Theory and experiment, AIAA Journal 22 (10) (1984) 1405-1412. doi: $10.2514 / 3.8796$.

URL http://arc.aiaa.org/doi/10.2514/3.8796

[7] M. J. Mandell, I. Katz, Theory of Hollow Cathode Operation in Spot and Plume Modes, in: 30th International Electric Propulsion Conference, no. 27-29 June, Indianapolis, Indiana, USA, 1994, pp. AIAA-94-3134.

[8] I. Katz, B. M. Gardner, M. L. Mandell, G. A. Jongeward, M. Patterson, R. M. Myers, Model of Plasma Contactor Performance, Journal of Spacecraft and Rockets 34 (6) (1997).

[9] I. Katz, M. J. Mandell, M. Patterson, M. Domonkos, Sensitivity of Hollow Cathode Performance to Design and Operating Parameters, in: 35th AIAAIASME/SAE/ASEE Joint Propulsion ccnterence \& Exhibit, no. 2023 July, Los Angeles, California, USA, 1999, pp. AIAA-99-2576.

[10] M. Capacci, M. Minucci, A. Severi, Simple numerical model describing discharge parameters in orificed hollow cathode devices, in: 33rd Joint Propulsion Conference and Exhibit, Joint Propulsion Conferences, no. 6-9 July, Seattle, Washington, USA, 1997. doi:https://doi.org/10.2514/ $6.1997-2791$.

[11] M. T. Domonkos, Evaluation of low-current orificed hollow cathodes, Ph.D. thesis, University of Michigan (1999).

[12] M. T. Domonkos, A Particle and Energy Balance Model of the Orificed Hollow Cathode, in: 38th AIAA/ASME/SAE/ASEE Joint Propulsion Conference \& Exhibit, no. 7-10 July, Indianapolis, Indiana, USA, 2002, pp. AIAA 2002-4240. doi:10.2514/6.2002-4240.

[13] D. M. Goebel, I. Katz, Fundamentals of Electric Propulsion - Ion and Hall Thrusters, John Wiley and Sons, Hoboken, New Jersey, 2008.

[14] I. Katz, J. Anderson, J. Polk, J. Brophy, One Dimensional Hollow Cathode Model, Journal of Propulsion and Power 19 (4) (2003) 595-600. doi: $10.2514 / 2.6146$.

[15] J. Mizrahi, V. Vekselman, V. Gurovich, Y. E. Krasik, Simulation of Plasma Parameters During Hollow Cathodes Operation, Journal of Propulsion and Power 28 (5) (2012) 1134-1137. doi:10.2514/1.B34406.

URL http://arc.aiaa.org/doi/10.2514/1.B34406 
[16] R. Albertoni, D. Pedrini, F. Paganucci, M. Andrenucci, A Reduced-Order Model for Low-Current Orificed Hollow Cathodes, IEEE Transactions on Plasma Science 41 (7) (2013) 1731-1745.

URL http://www.academia.edu/download/30451697/SPC2012\{_\} 2349890.pdf

[17] I. G. Mikellides, I. Katz, D. M. Goebel, J. E. Polk, Hollow cathode theory and experiment. II. A two-dimensional theoretical model of the emitter region, Journal of Applied Physics 98 (11) (2005) 1-14. doi:10.1063/1. 2135409.

[18] I. G. Mikellides, I. Katz, D. M. Goebel, J. E. Polk, Model of a Hollow Cathode Insert Plasma, in: 40th AIAA/ASME/SAE/ASEE Joint Propulsion Conference \& Exhibit, no. 11-18 July, Fort Lauderdale, Florida, USA, 2004, pp. AIAA 2004-3817. doi:10.2514/6.2005-4234.

[19] C. J. Wordingham, P.-Y. Taunay, E. Choueiri, A Critical Review of Orificed Hollow Cathode Modeling, in: 53rd AIAA/SAE/ASEE Joint Propulsion Conference, no. 10-12 July, Atlanta, Georgia, USA, 2017, pp. AIAA-20074888. doi:10.2514/6.2017-4888.

URL https://arc.aiaa.org/doi/10.2514/6.2017-4888

[20] W. Schottky, Concerning the Discharge of Electrons from Hot Wires with Delayed Potential, Annalen der Physik 349 (15) (1914) 1011-1032.

[21] G. J. Williams, T. B. Smith, M. T. Domonkos, A. D. Gallimore, R. Paul Drake, Laser-induced fluorescence characterization of ions emitted from hollow cathodes, IEEE Transactions on Plasma Science 28 (5) (2000) 16601663. doi:10.1109/27.901251.

[22] G. Sary, L. Garrigues, J.-P. Boeuf, Hollow cathode modeling: II. Physical analysis and parametric study, Plasma Sources Science and Technology 26 (5) (2017) 55008. doi:10.1088/1361-6595/aa6210.

URL http://stacks.iop.org/0963-0252/26/i=5/a=055008

[23] J. M. Lafferty, Foundations of vacuum science and technology, John Wiley \& Sons, Inc, 1998.

[24] A. Roth, Vecuum technology, Elsevier B.V., Amsterdam, 1990.

[25] I. G. Mikellides, I. Katz, Wear Mechanisms in Electron Sources for Ion Propulsion, I: Neutralizer Hollow Cathode, Journal of Propulsion and Power 24 (4) (2008) 855-865. doi:10.2514/1.33461.

URL http://arc.aiaa.org/doi/10.2514/1.33461

[26] J. G. Andrews, J. E. Allen, Theory of a Double Sheath between Two Plasmas, Proceedings of the Royal Society of London. Series A, Mathematical and Physical Sciences 320 (1543) (1971) 459-472. 
[27] F. W. Crawford, J. L. Freeston, Negative Ion Sources: Magnetron and Penning, in: 6th International Conference of lonization Phenomena in Gases, Paris, France, 1963.

[28] M. A. Lieberman, A. J. Lichtenberg, Principles of Plasma Discharges and Material Processing, 2nd Edition, Vol. 53, John Wiley \& Sons, Inc, Hoboken, 2005. arXiv:arXiv:1011.1669v3.

[29] J. A. Bittencourt, Fundamentals of Plasma Physics, 3rd Edition, Vol. 72, Springer Science+Business Media, LLC, New York, NY, 2004.

[30] I. G. Mikellides, I. Katz, M. J. Mandell, J. S. Snyder, S. S. Loral, A 1-D Model of the Hall-Effect Thruster with an Exhaust Region, in: 37th AIAA / ASME / SAE / ASEE Joint Propulsion Conference and Exhibit, no. 8-11 July, Salt Lake City, Utah, USA, 2001, pp. AIAA-2001-3505.

[31] J. L. Cronin, Modern dispenser cathodes, in: IEEE Proceedings I (Solid State and Electron Devices), Vol. 128, 1981, pp. 19-32. doi:10.1049/ ip-i-1.1981.0012.

[32] P. D. Prewett, J. E. Allen, The Double Sheath Associated with a Hot Cathode, Proceedings of the Royal Society of London. Series A, Mathematical and Physical Sciences 348 (1655) (1976) 435-446.

[33] D. Pedrini, R. Albertoni, F. Paganucci, M. Andrenucci, Theoretical model of a lanthanum hexaboride hollow cathode, IEEE Transactions on Plasma Science 43 (1) (2015) 209-217. doi:10.1109/TPS.2014. 2367815.

[34] D. Courtney, Development and characterization of a diverging cusped field thruster and a lanthanum hexaboride hollow cathode, Ph.D. thesis, Massachusetts Institute of Technology (2008).

URL http://dspace.mit.edu/handle/1721.1/45239

[35] D. Goebel, E. Chu, High Current Lanthanum Hexaboride Hollow Cathodes for High Power Hall Thrusters, in: 32nd International Electric Propulsion Conference, no. 11-15 Septembe, Wiesbaden, Germany, 2011, pp. IEPC2011-053. doi:10.2514/1.B34870.

[36] G.-c. Potrivitu, L. Xu, I. Levchenko, S. Huang, Y. Sun, M. Wisnuh, R. Aggriawan, J. Wei, M. Lim, K. Bazaka, S. Xu, Mode Transition in a Lowcurrent LaB6 Hollow Cathode for Electric Propulsion Systems for Small Satellites, 36th International Electric Propulsion Conference (2019) IEPC$2019-427$.

[37] A. Lucca Fabris, C. V. Young, M. A. Cappelli, Excited state population dynamics of a xenon ac discharge, Plasma Sources Science and Technology 24 (5) (2015) 055013 (11pp). doi:10.1088/0963-0252/24/5/055013. 